\title{
Impact of a computer-based breastfeeding education program on breastfeeding self-efficacy and duration in rural Hispanic women
}

\author{
Trina M Aguirre ${ }^{1 *}$, Ashish Joshi ${ }^{2}$, Ann E Koehler ${ }^{1}$, Eli K Rodriguez ${ }^{1}$ and Susan L Wilhelm ${ }^{1}$ \\ ${ }^{1}$ College of Nursing - West Nebraska Division, University of Nebraska Medical Center, Scottsbluff, NE, USA \\ ${ }^{2}$ Population Health Informatics, Graduate School of Public Health and Health Policy, City University of New York, New York, USA
}

\begin{abstract}
Background: Breastfeeding provides numerous benefits for both infant and mother. However, duration of breastfeeding does not meet Healthy People 2020 goals.

Research Aim: Researchers developed and evaluated a bilingual, computer-based breastfeeding education program to promote breastfeeding by rural Hispanic mothers. This secondary analysis further examined breastfeeding self-efficacy and duration.

Methods: This study was conducted using a two-group repeated measures quasi-experimental design. Participants were randomly assigned to receive breastfeeding education through our computer-based program (intervention, $\mathrm{n}=23$ ) or printed materials (control, $\mathrm{n}=23$ ). Assessments were performed at baseline, 3 and 7 days, 2 and 6 weeks, 3 and 6 months postpartum.

Results: Duration of breastfeeding did not differ between mothers in the intervention and control groups $(p=0.962)$. The greatest decline in breastfeeding occurred between weeks 2 and 6 and months 3 and 6. Top reasons for discontinuing breastfeeding were issues with milk supply, returning to work, and latch. Mothers who were breastfeeding tended to have greater self-efficacy scores than mothers who were not, significantly so at week 6 and months 3 and 6 ( $p<0.001$ ). Intervention mothers using the program tended to breastfeed longer and a greater proportion were still breastfeeding at 6 months, however, program usage was light.

Conclusion: Our bilingual computer-based breastfeeding education support program showed potential as a means to improve breastfeeding self-efficacy in this rural Hispanic population. Future efforts will need to take steps to improve program use, perhaps by adapting the program for smartphone delivery and introducing the program to mothers prenatally.
\end{abstract}

The parent RCT study was registered (\# NCT03308058) with Clinical Trials.gov on October 30, 2012.

\section{Background}

Breastfeeding is the most important first health promotion strategy available for infants and provides many benefits for both mother and child [1]. The American Academy of Pediatrics [2] recommends breastfeeding exclusively for the first 6 months, continuing with human milk as the primary food for at least 12 months. In the United States, national goals are to have $81.9 \%$ of infants ever breastfed and $60.6 \%$ breastfed for six months $(25.5 \%$ exclusively) by the year 2020 [3-5] Currently, about $81 \%$ of U.S. infants are breastfed immediately postpartum. However, by 6 months just over half (52\%) receive any human milk with only $22 \%$ breastfed exclusively [6]. Rates of exclusive breastfeeding tend to be lower for Hispanic mothers because supplementation is common, even within the first week after birth [7].

Human milk provides optimal nutrition for the healthy growth and mental and physical development of children and is protective against a variety of diseases (e.g. gastrointestinal illness, otitis media, and respiratory infections) $[1,8]$. Furthermore, greater duration/exclusivity of breastfeeding is associated with higher intelligence test scores, which may impact future academic performance [9]. Breastfed infants also experience less diabetes and obesity, conditions that tend to be more prevalent in rural and Hispanic populations [10].
Cultural attitudes/beliefs can influence breastfeeding practices. Although most Hispanic-American mothers initiate breastfeeding (84\%), duration and exclusivity remain low (only $48 \%$ still breastfeeding at 6 months, $17 \%$ exclusively) [3-5]. Many Hispanics consider chubby infants to be healthy, introduce solid foods early, and feed both human milk and formula (los dos), believing it provides the best nutrition and ensures against hunger $[7,11]$. Modesty about breastfeeding in public is also a factor.

Breastfeeding rates also differ with place of residence and tend to be lower among rural than urban residents [12]. A contributing factor may be that rural residents are often disadvantaged in obtaining care because of a shortage of health care professionals (e.g. in Nebraska, $62 \%$ of the counties are designated as family practice shortage areas and $86 \%$ are psychiatry/mental health shortage areas) [13]. In the Rural Healthy People 2020 Survey, rural residents identified access to health care as their top concern [14]. Transportation issues also factor into access, particularly in families with limited income [15].

${ }^{\star}$ Correspondence to: Trina M. Aguirre, College of Nursing - West Nebraska Division, University of Nebraska Medical Center, 1601 East 27th, Scottsbluff, NE 69361-1815, USA, Telephone: (308) 632-0429; Email: taguirre@unmc.edu

Received: July 05, 2018; Accepted: July 20, 2018; Published: July 23, 2018 
To address these issues, our research team developed and evaluated an interactive, bilingual, computer-based breastfeeding educational support program to promote breastfeeding by Hispanic (primarily Mexican-American) women in rural western Nebraska, USA. Here we report the results of a secondary analysis that further examined breastfeeding self-efficacy, duration of breastfeeding, and considerations for future directions.

\section{Research Aims}

\section{Duration (Days) of Breastfeeding}

We explored patterns of continuing/discontinuing breastfeeding and mothers' reasons for stopping breastfeeding. Specific aims were to:

1. Compare duration of breastfeeding among mothers in the intervention and control groups

2. Explore when mothers discontinued breastfeeding relative to the study time points ( 3 and 7 days, 2 and 6 weeks, and 3 and 6 months postpartum) and

3. Explore mothers' self-reported reasons for discontinuing breastfeeding.

\section{Breastfeeding Self-efficacy}

This exploration of breastfeeding self-efficacy primarily focused on the relationship between breastfeeding self-efficacy and duration of breastfeeding. Specific aims were to:

1. Compare the breastfeeding self-efficacy scores of mothers (either group) who were still breastfeeding at 6 months versus the scores of those who were not and

2. Compare the breastfeeding self-efficacy scores of mothers (either group) who were or were not breastfeeding at each time point.

\section{Program Use (Intervention Group)}

Additionally, we explored patterns of program use (self-report) in relation to duration of breastfeeding and examined participantvolunteered comments to better understand factors influencing use. Specific aims were to:

\section{Examine program use (yes/no) at each time point}

2. Examine total use over the 6-month study (number of yes responses over all time points)

3. Compare duration of breastfeeding between intervention mothers who did and did not use the program and

4. Explore self-reported reasons for not using the program.

\section{Methods}

\section{Design}

This secondary analysis focused on a subset of data from a twogroup repeated measures quasi-experimental study that evaluated the impact of an interactive, bilingual, computer-based breastfeeding educational support program to promote breastfeeding among rural Hispanic women. This design enabled us to compare breastfeeding selfefficacy and duration between mothers receiving the computer-based breastfeeding education intervention and those receiving the attention control over the recommended minimum period for breastfeeding ( 6 months).
Participants were randomly assigned to receive bilingual breastfeeding education through either the computer-based program (intervention, $\mathrm{n}=23$ ) or through printed materials (attention control, $\mathrm{n}=23$ ). Those in the intervention group received the web-address for the computer-based program and all participants received the printed educational materials. Participants accessed the educational content at their convenience as desired. Assessments were completed at baseline, 3 and 7 days, 2 and 6 weeks, and 3 and 6 months postpartum. Postpartum assessments were scheduled at time points when mothers are particularly susceptible to discontinuing breastfeeding.

This study was conducted in accordance with IRB protocols 43012-EP (University of Nebraska Medical Center) and 642980-1 (City University of New York). Those choosing to participate were consented and assigned an ID number to maintain confidentiality.

\section{Setting}

This study was conducted in the Nebraska Panhandle, a rural region with an average population density of 6 people per mile ${ }^{2}[16]$ and a large, well-established Hispanic, primarily Mexican-American, population. Research activities were based in Scottsbluff, Nebraska at University of Nebraska Medical Center (UNMC) and Regional West Medical Center (RWMC) facilities. Data were collected between August 30, 2013 and August 21, 2014. An individual participant's involvement was the first 6 months postpartum.

\section{Sample}

This study targeted rural Hispanic women who intended to breastfeed their infants. Participants were recruited through the Women's Center and the Birth and Infant Care Center at RWMC, a regional referral hospital, during their third trimester or shortly after delivery. RWMC staff informed women meeting the inclusion criteria (self-described Hispanic females $\geq 15$ years old who were able to use/had access to the internet, and were available for follow-up assessments) about the study. Those who expressed interest were asked for permission to release their names to the researchers.

The sample size for this study was determined through a comparison of two independent binomial proportions using Pearson's Chi-square statistic with a two-sided significance level of 0.05 which indicated that a sample size of 23 per group achieved a power of at least 0.8 when the proportion with partial breastfeeding at 6 months of 0.3 for the control group and 0.7 for the intervention group. Fifty-eight potential participants were contacted by the researchers, 46 were consented and enrolled (23 per group), and 12 were excluded because of ineligibility. No participants were lost to attrition [15].

\section{Measurement}

This secondary analysis further explored duration of any breastfeeding, breastfeeding self-efficacy, program use (intervention group), and mothers' self-offered comments.

Duration of breastfeeding was calculated as the number of days between initiating and ending breastfeeding based on mothers' selfreport. If a mother discontinued breastfeeding, the date she stopped and her reasons were reported on the breastfeeding assessment questionnaire (questions about the mother's experiences with breastfeeding including feeding patterns, problems encountered, supplementation, complementary feeding, returning to work, etc.) that was completed at each follow-up assessment).

Self-efficacy (a mother's confidence in her ability to breastfeed) was measured using the 14-item Breastfeeding Self-Efficacy Scale-Short 
Form (BSES-SF) that was scored using a 5-point Likert-type scale [17]. The self-efficacy score (possible range 14-70) was the sum of the scores for each item with higher scores indicating greater confidence. The BSES-SF demonstrated internal consistency (Cronbach's $\alpha=0.94$ ) and predictive validity at 4 and 8 weeks postpartum [17].

Data on program use (intervention group) were compiled from notes recorded at each assessment time point. These data include the mother's response (yes or no) when asked whether she had used the program since the last time point and any comments the mother volunteered regarding difficulties encountered and reasons for not using the program.

To accommodate language preferences, all study materials were available in both English and Spanish and a certified translator (bilingual promotora) was available throughout the study to assist as needed. Participants received a $\$ 15$ Target gift card incentive following each assessment to compensate them for their time.

\section{Data Collection}

Women who expressed interest in the study were visited by a CITI-trained research staff member (the senior author or a bilingual promotora) who described the study (verbally and with written materials) and invited them to participate. Those choosing to do so signed a written consent.

Following consenting, participants completed a set of baseline questionnaires. Follow-up assessment questionnaires were completed 3 and 7 days, 2 and 6 weeks, and 3 and 6 months postpartum via the mother's choice of telephone interviews with the senior author or promotora, or by completing hard copies of the forms delivered/picked up by the researchers). Contacts were initiated by the senior author or promotora at times convenient for the participants.

\section{Data Analyses}

All data analyses were performed using IBM $^{\circ}$ SPSS ${ }^{\circ}$ Statistics (Version 25) software. Descriptive statistics (e.g. means, standard deviations, frequencies, percentages) were used to characterize variables/responses and independent T-tests (2-tailed) were used to explore differences between groups $(\alpha=0.05)$.

\section{Results}

\section{Duration of Breastfeeding}

Duration of breastfeeding did not differ between mothers in the intervention $(M=121.2, S D=65.9$ days, range $=18-180$ days $)$ and control $(M=120.3, S D=61.8$ days, range $=4-180$ days $)$ groups $(p=0.962)$. Both groups also showed similar patterns of decline in breastfeeding over time with the greatest decline occurring between weeks 2 and 6 and months 3 and 6 (Figure 1), suggesting that women in this population are particularly at risk for discontinuing breastfeeding during these time intervals.

\section{Reasons for Discontinuing Breastfeeding}

The top three reasons cited for discontinuing breastfeeding were insufficient milk supply $(\mathrm{n}=12)$, work/school commitments $(\mathrm{n}=10)$, and issues establishing latch $(\mathrm{n}=5)$. This was true for both intervention $(n=6,5,3$, respectively) and control mothers $(n=6,5,2$, respectively). These responses were self-reported with some mothers citing multiple reasons for discontinuing breastfeeding and others reporting none.

\section{Breastfeeding Self-efficacy}

Breastfeeding self-efficacy did not differ between intervention and control mothers overall [18] or by time point [19]. Further exploration of self-efficacy revealed that mothers who were still breastfeeding at 6 months (either group) tended to have greater self-efficacy scores than mothers who had discontinued breastfeeding. Though scores did not differ between these mothers at baseline or the early days postpartum, differences in self-efficacy scores were significant at week 6 and months 3 and $6(p<0.001)$ (Table 1$)$. These findings suggest that increasing women's breastfeeding self-efficacy may help them breastfeed longer.

\section{Exploration of Intervention Responses}

We explored reasons for the lack of difference in breastfeeding selfefficacy/duration between intervention and control groups by delving deeper into our data, including participant comments.

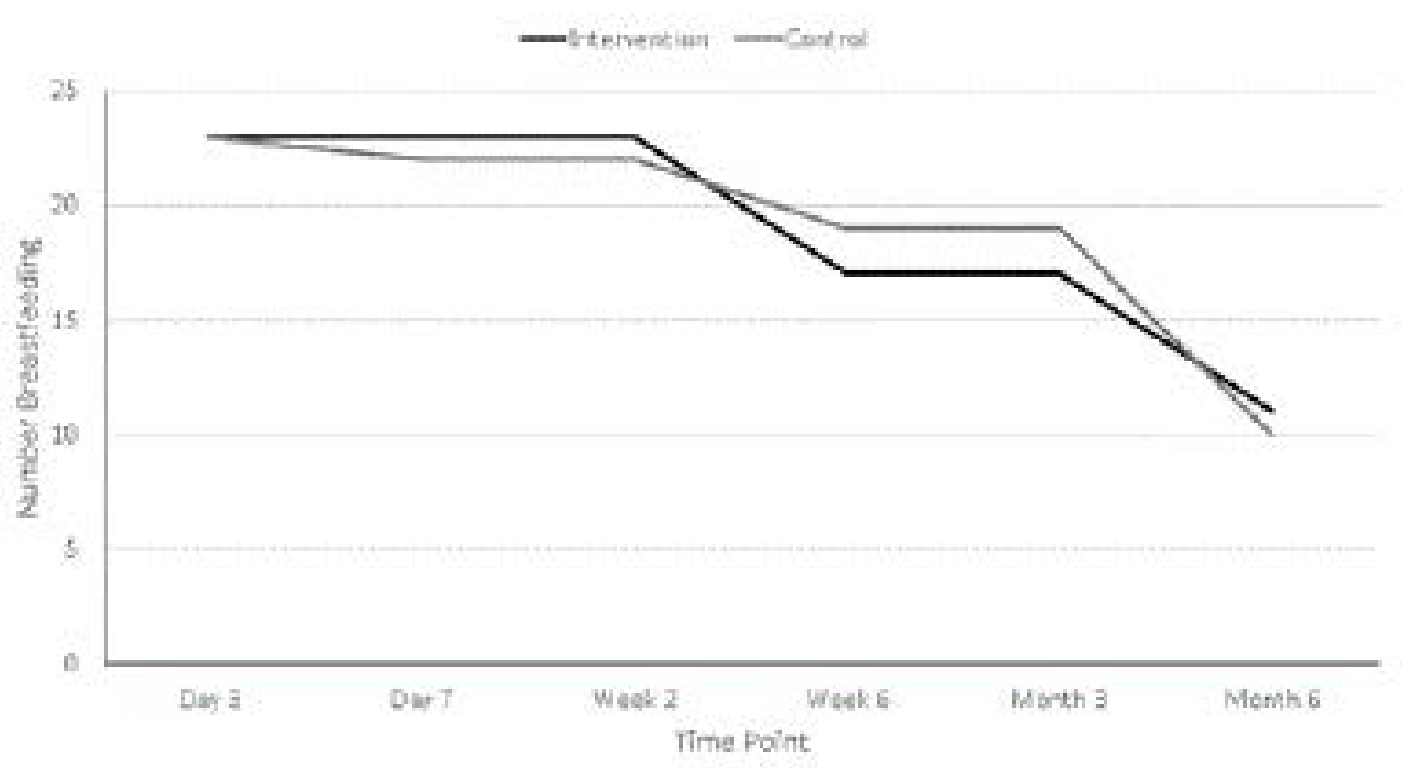

Figure 1. Number of women in each group who were breastfeeding at each time point (Intervention $n=23$, control $n=23$ ) 
Table 1. Comparison of Mean $( \pm \mathrm{SD})$ Breastfeeding Self-efficacy Scores of Mothers who were and were not Breastfeeding at 6 Months

\begin{tabular}{|c|c|c|c|c|}
\hline \multirow{2}{*}{ Time } & $\begin{array}{c}\text { Breastfeeding } \\
\mathbf{n = 2 1}\end{array}$ & $\begin{array}{c}\text { Discontinued } \\
\mathbf{n = 2 5}\end{array}$ & $\boldsymbol{t}$ & $\boldsymbol{t}$ \\
\cline { 2 - 4 } & $\boldsymbol{M}(\boldsymbol{S D})$ & $\boldsymbol{M}(\boldsymbol{S D})$ & & 0.44 \\
\hline Baseline & $56.2(12.2)$ & $53.7(9.5)$ & 0.78 & 0.69 \\
\hline Day 3 & $61.4(7.3)$ & $60.3(10.7)$ & 0.40 & 0.08 \\
\hline Day7 & $63.2(6.6)$ & $57.1(15.5)$ & 1.80 & 0.06 \\
\hline Week 2 & $63.9(6.1)$ & $58.8(11.0)$ & 1.96 & $<0.001$ \\
\hline Week 6 & $66.2(5.7)$ & $54.4(12.8)$ & 4.15 & $<0.001$ \\
\hline Month 3 & $66.5(4.8)$ & $53.1(13.1)$ & 4.66 & $<0.001$ \\
\hline Month 6 & $67.1(4.0)$ & $53.8(13.2)$ & 4.52 & \\
\hline
\end{tabular}

\section{Intervention program usage by assessment interval}

Overall, self-reported program use was light (range $=14-39 \%$ of moms who were still breastfeeding at each time point) and was greater in the early days and weeks postpartum, peaking at weeks $2(35 \%)$ and $6(39 \%)$. Concerning is that over one third of intervention mothers $(35 \%, n=8)$ did not use the program after they were enrolled. The mean number of time points mothers reported using the program was 1.4 $(S D=1.4$, range $=0-4$ times $)$ overall and $2.1(S D=1.3$, range $=1-4$ times $)$ for those who used the program after enrollment $(n=15)$.

\section{Breastfeeding duration by program usage}

Though breastfeeding duration did not differ between intervention and control groups overall, intervention mothers who used the program tended to breastfeed longer $(M=126.4, S D=63.7$, range $=26-180$ days, $\mathrm{n}=15)$ than those who $\operatorname{did} \operatorname{not}(M=111.5, S D=73.3$, range $=18-180, \mathrm{n}=8)$ and a greater proportion were still breastfeeding at 6 months $(53.3 \%$; $\mathrm{n}=8$ of 15$)$ compared to those not using the program $(37.5 \% ; \mathrm{n}=3$ of 8). Intervention mothers who were breastfeeding at 6 months tended to use the program more $(M=1.9, S D=1.6$, range $=0-4$ times, $n=11)$ than those who discontinued breastfeeding before 6 months $(M=0.8$, $S D=1.1$, range $=0-4$ times, $\mathrm{n}=12$ ).

\section{Reasons for not using the program}

Most mothers did not offer a reason for not using the program. Among those who did, the top reasons cited were access to a computer/ the internet (reported by 1-4 mothers per time point), losing the website address (reported by 1-2 mothers per time point), and problems accessing/using the program (reported by 1-2 mothers per time point). Only one mother reported not accessing the program because of time constraints and only on one occasion.

\section{Discussion}

Many women in this population do not participate in prenatal classes $[17,20]$. Based on our earlier pilot studies, use of computer/ internet-based multimedia technology showed promise as a strategy for providing health information, to rural Hispanics [21,22]. Therefore, we developed and evaluated an interactive, bilingual, computer-based breastfeeding educational support program as a means of providing breastfeeding education to these rural Hispanic women.

\section{Duration of Breastfeeding}

Results of this secondary analysis revealed that the greatest decline in breastfeeding occurred between weeks 2 and 6 and months 3 and 6 postpartum in both the intervention and control groups. Mothers may discontinue breastfeeding at 2 weeks because they perceive their milk supply to be insufficient and at 6 weeks because many return to work [23]. These were the primary reasons cited by the nine women who discontinued breastfeeding between weeks 2 and 6 in this study (milk supply $n=4$, work $n=3$ ). Formula supplementation is common in this population and may have contributed to mothers' perceptions of insufficient milk supply as this practice inhibits breast milk production [7]. In this study, supplementation began early and was common throughout. Among mothers breastfeeding at each time point, 40$50 \%$ were supplementing through week 6 and approximately one third were supplementing at months 3 and 6, well above the Healthy People 2020 [24] target of no more than $14.2 \%$ of newborn infants receiving supplementation within two days postpartum.

Women who discontinued breastfeeding between 3 and 6 months $(n=15)$ cited few reasons for stopping (milk supply $n=1$, work $n=1$, baby preferred bottle $n=2$, mother's health $n=1$ ). Though not cited as a reason for discontinuing breastfeeding, all but 7 of the 46 mothers reported introducing solid foods between 3 and 6 months (overall $M=5.5, S D=0.9$ months, intervention $M=4.9, S D=0.9$ months, control $M=5.2, S D=0.8$ months). Perhaps some mothers began weaning their children during the interval between 3 and 6 months, contributing to the decline in mothers who were still breastfeeding.

\section{Breastfeeding Self-efficacy}

Results from these analyses suggest that suggest that increasing women's breastfeeding self-efficacy may help them breastfeed longer and that mothers in the intervention group who used the program tended to have longer duration of breastfeeding than those who did not.

\section{Program Usage (Intervention Group)}

Concerning was the finding that program usage was light during this study and that over a third of intervention mothers did not use the program after enrollment. Issues affecting program use (e.g. access to a computer/the internet, losing the website address, problems accessing/ using the program) need to be addressed.

Anytime a mother lost the website address, it was resent to her as soon as possible. The problem of accessing the computer/internet was a little surprising because at baseline, all but one of the intervention mothers reported having a computer at home/using the internet with most mothers reporting that they use the computer/internet daily or weekly (computer $70 \%$, internet $78 \%$ ). Several mothers (both intervention and control) volunteered that they accessed the internet through their smartphones. Specific issues included broken computer/ smartphone, no longer having a computer, or for one mother, the library where she used the computer was under renovation.

\section{Future Implications}

The observed trends suggest that our bilingual computer-based breastfeeding support program has potential to improve breastfeeding duration by rural Hispanic mothers if program use can be improved. One approach might be to adapt the program for use via smartphones as many in this population use their phones to access the internet. Potential limitations of this approach are that other household members may control use of smartphone minutes and, in lower income families, minutes are only added when money is available [15]. Also, it may be helpful to encourage mothers to use the program prenatally, as they may become overwhelmed by the lifestyle changes during the first days following birth.

Given the reasons stated for discontinuing breastfeeding, topics that may need additional emphasis include supplementation, milk supply, and the trade-off between the two. Some supplementation may occur because of mothers' concerns about whether their baby is 
getting enough to eat, therefore, content needs to include approaches enabling mothers to confidently recognize that their baby is getting enough. In this population, the practice of los dos [7] and the cultural preference for heavier babies [24] contribute to the prevalence of supplementation and need to be addressed with sensitivity. Another area of emphasis should be on preparing to return to work/balancing work and breastfeeding.

\section{Limitations}

These secondary analyses take advantage of data originally collected to meet other objectives and include analyses of participantvolunteered information which may not reflect the views/experiences of all participants. Results of these secondary analyses and the information participants chose to share did, however, provide insights that can aid in the development of future interventions/research studies.

\section{Conclusions}

Our bilingual computer-based breastfeeding support program showed potential as a means to improve breastfeeding self-efficacy and duration by rural Hispanic mothers, however, we need to improve the level of use, perhaps by adapting the program for smartphone delivery and introducing the program to mothers prenatally. Enhancing the content to better address common reasons for discontinuing breastfeeding (e.g. milk supply, returning to work) and associated issues could also improve the program's value for new mothers.

\section{Disclosure statement}

Conflicts of Interest: None.

\section{Funding}

This study was supported by the Agency for Healthcare and Research Quality (AHRQ) under grant \#HS02132101A1.

\section{Acknowledgements}

The authors thank Lupe Salazar and Mary Ann Shockley for translation and interpretation services during the conduct of this study.

\section{References}

1. AAFP (2014) Breastfeeding, Family Physicians Supporting (Position paper). American Academy of Family Physicians.

2. American Academy of Pediatrics (2012) Policy on breastfeeding and use of human milk. Pediatrics 129: e827-e841.

3. Healthy People (2020a) Maternal, Infant, and Child Health. MICH-21.1 Increase the Proportion of Infants who are ever Breastfed. U.S. Department of Health and Human Services (n.d.).

4. Healthy People (2020b) Maternal, Infant, and Child Health MICH-21.2 Increase the Proportion of Infants who are Breastfed at 6 Months. U.S. Department of Health and Human Services (n.d.)
5. Healthy People (2020c) Maternal, Infant, and Child Health MICH-21.5 Increase the Proportion of Infants who are Breastfed Exclusively Through 6 Months. U.S. Department of Health and Human Services (n.d.)

6. CDC (2016) Breastfeeding report card, Centers for Disease Control and Prevention.

7. Sloand E, Budhathoki C, Junn J, Vo D, Lowe V, et al. (2016) Breastfeeding among Latino families in an urban pediatric office setting. Nursing Research and Practice.

8. WHO (2013) Essential Nutrition Actions: Improving Maternal, Newborn, Infant And Young Child Health And Nutrition. World Health Organization.

9. Horta B, Loret de Mola C, Victora C (2015) Breastfeeding and intelligence: A systematic review and meta-analysis. Acta Paediatrica 104: 14-19.

10. Modrek S, Basu S, Harding M, White JS, Bartick MC, et al. (2017) Does breastfeeding duration decrease child obesity? An instrumental variables analysis. Pediatr Obes 12: 304-311. [Crossref]

11. Cartagena DC, Ameringer SW, McGrath J, Jallo N, Masho SW, et al. (2014) Factors contributing to infant overfeeding with Hispanic mothers. J Obstet Gynecol Neonatal Nurs 43: 139-159.

12. Aschbrenner K, Lubker-Cornish D (2017) Barriers to breastfeeding among rural women in the United States. Universitas 12: 2016-2017.

13. Nebraska Department of Health and Human Services (2016) Nebraska Rural Health Advisory Commission State Designated Shortage Areas - Medical and Mental Health.

14. Bolin JN, Bellamy GR, Ferdinand AO, Vuong AM, Kash BA, et al. (2015) Rural Healthy People 2020: New Decade, Same Challenges. J Rural Health 31: 326-333. [Crossref]

15. Aguirre T, Koehler A, Joshi A, Wilhelm S (2016) Recruitment and retention challenges and successes. Ethnicity \& Health 22: 1-9.

16. PADD (2014) Panhandle Area Development District, Nebraska Panhandle Area Development District.

17. Dennis C (2003) The breastfeeding self-efficacy scale: Psychometric assessment of the short form. Journal of Obstetric \& Gynecological Neonatal Nursing 32: 734-744.

18. Joshi A, Amadi C, Meza J, Aguirre T, Wilhelm S (2015) Comparison of sociodemographic characteristics of a computer based breastfeeding educational intervention among rural Hispanic women. J Community Health 40: 993-1001.

19. Joshi A, Amadi C, Meza J, Aguirre T, Wilhelm S (2016) Evaluation of a computerbased bilingual breastfeeding educational program on breastfeeding knowledge, selfefficacy and intent to breastfeed among rural Hispanic women. Int J Med Inform 91: 10-19.

20. Joshi A, Trout KE, Aguirre T, Wilhelm S (2014) Exploration of factors influencing initiation and continuation of breastfeeding among Hispanic women living in rural settings: A multi-methods study. Rural Remote Health 14: 2955.

21. Aguirre T, Wilhelm S, Joshi A (2012) Assessment of technology access and preference for health education of a rural Hispanic community. Technol Health Care 20: 551-555.

22. Wilhelm S, Aguirre T, Koehler A, Rodehorst T (2015) Evaluating motivationa interviewing to promote breastfeeding by rural Mexican-American mothers: The challenge of attrition. Issues Compr Pediatr Nurs 38: 7-21.

23. Healthy People (2020d) Maternal, Infant, and Child Health MICH-23 Reduce the Proportion of Breastfed Newborns Who Receive Formula Supplementation within the First 2 Days of Life. U.S. Department of Health and Human Services (n.d.)

24. Franzen-Castle L, Aguirre T (2015) Perceptions of Body Habitus and Cultural Health Among Hispanic Adults. J Immigr Minor Health 17: 1206-1213. [Crossref]

Copyright: (C2018 Aguirre TM. This is an open-access article distributed under the terms of the Creative Commons Attribution License, which permits unrestricted use, distribution, and reproduction in any medium, provided the original author and source are credited. 